\title{
A Perturbed Half-normal Distribution and Its Applications
}

\author{
Eisa Mahmoudi*, Reihaneh Lalehzari and Rahmat Sadat Meshkat \\ Yazd University
}

Received: $12 / 23 / 2016 \quad$ Approved: $1 / 21 / 2018$

\begin{abstract}
In this paper, a new generalization of the half-normal distribution which is called the perturbed half-normal distribution is introduced. The new distribution belongs to a family of distributions which includes the half-normal distribution along with an extra parameter to regulate skewness. The probability density function (pdf) is derived and some various properties of the new distribution are obtained. The derived properties include the cumulative distribution function (cdf), the $r$ th moment, moment generating function, characteristic function, mean deviation about the mean and estimation of the parameters using the method of moments and maximum likelihood. Finally, the flexibility and potentiality of the new distribution is illustrated in an application to two real data sets.
\end{abstract}

Keywords. Error function; half-normal distribution; hypergeometric function; skewness; moment generating function.

MSC 2010: 60E05, 62H10, 62H12.

\section{Introduction}

The half-normal (HN) distribution may be an initial choice in modeling monotone hazard rate functions because of its negatively and positively

\footnotetext{
* Corresponding author
}

Copyright $₫$ 2019, ASP Ins. This open-access article is published under the terms of the Creative Commons AttributionNonCommercial 4.0 International License which permits Share (copy and redistribute the material in any medium or format) and Adapt (remix, transform, and build upon the material) under the Attribution-NonCommercial terms. 
skewed density shapes. The HN distribution has applications in various contexts, particularly in areas such as blowfly dispersion (Dobzhansky and Wright, 1947), interpreting factorial two-levels experiments (Daniel, 1959), stochastic frontier modeling (Aigner et al., 1977; Meeusen and van den Broeck, 1977), diverse as fiber buckling (Haberle, 1991), describe the lifetime process under fatigue (Mitchell and Landgraf, 1996), quality control (Chou and Liu, 1998), dealing with relationships between measurement error and magnitude (Bland and Altman, 1999) and sport science physiology (Pewsey, 2002, 2004).

Pewsey $(2002,2004)$ considered the likelihood based inference for the original parametrization of the general HN distribution. Wiper et al. (2008) considered approaches to Bayesian inference for the HN and half- $t$ distributions using uninformative priors. They showed that exact Bayesian inference can be undertaken for the half-normal distribution without the need for Gibbs sampling.

Recently, authors have been attempted to define new families of probability distributions that extend well-known families of distributions and at the same time provided great flexibility in modeling data in practice. Different methods may be used to add a shape parameter to a HN distribution. For example, Pescim et al. (2009) proposed a new family of beta generalized half-normal distribution based on the kernel of beta distribution and Cooray and Ananda (2008) proposed the generalized half-normal (GHN) distribution derived from a model for static fatigue. These two models extend the $\mathrm{HN}$ distribution by adding a shape parameter.

An approach to introduce a new distribution as extension of a specific distribution is using the formula $c(\lambda) f(x) F(\lambda x)$. If $f(x)$ is symmetric about zero, then $c(\lambda)=2$ and if $f(x)$ is non-symmetric, then $c(\lambda)=E[F(\lambda X)]^{-1}$. Azzalini (1985) was characterized the skew normal distribution with replacing $f$ and $F$ by pdf and cdf of standard normal distribution. Using this approach to generalize distributions with positive support is not received considerable attention in literature. In this paper, by substituting pdf and cdf of half-normal distribution, we introduce a new generalization of the half-normal distribution called as perturbed half-normal (PHN) distribution which is completely different from the previous methods. Parameter $\lambda$ governs the shape of the probability distribution function of the new distribution. HN distribution is a especial case of PHN distribution as $\lambda \rightarrow \infty$.

In the following we provide some important motivations for introducing this model: 
1. The PHN distribution provides a reasonable parametric fit for modelling phenomenon with increasing failure rates, that the HN distribution cannot be properly fitted.

2. This new generalization can be as a candidate model for describing the lifetime under fatigue processes.

3. Performing the PHN distribution instead of HN distribution derives more appropriate results in real applications such as (i) in the EGARCH specification for asymmetric volatility modelling (Radson, 1995), (ii) in quality control, the PHN can serve as the basis of the chart for the absolute value of successive differences. (iii) in Baysian statistics, the PHN distribution can be used as non-informative prior distribution on the standard deviation of standard distributions (Gelman, 2006).

The PHN distribution has a compact form and all the moments can be computed explicitly. Therefore, mean, variance, skewness, kurtosis, moment generating function (mgf), characteristic function and hazard rate function, all can be computed explicitly. The rest of the paper is organized as follows: In Section 2, we introduce the probability density function (pdf) of the PHN distribution. Section 3 is devoted to finding the cumulative distribution function (cdf) of the PHN distribution. Also, the hazard rate function and survival function are obtained in this section. Section 4 provides a general expansion for the moments of the PHN distribution. Its moment generating function and characteristic function are derived in this section. We investigate on some properties of the PHN distribution in Section 5. Mean deviation from the mean is obtained in Section 6. In Section 7, we discuss moments and maximum likelihood estimation and calculate the elements of the observed information matrix. Simulation study is given in Section 8 . Application of the PHN distribution to two real data sets is given in Section 9. Finally, Section 10 concludes the paper.

\section{The Perturbed Half-normal Distribution}

In this section, we introduce the perturbed half-normal (PHN) distribution, by applying the well-known method of Azzalini (1985) to the non-symmetric half-normal distribution.

Definition 1. A random variable $X$ is said to have the PHN distribution, 
with the shape parameter $\lambda>0$, if it's pdf is given by

$$
f(x ; \lambda)=c(\lambda) g(x) G(\lambda x), \quad x>0,
$$

where $g(x)$ and $G(x)$ are the pdf and cdf of the standard half-normal distribution specified by

$$
g(x)=\sqrt{\frac{2}{\pi}} e^{-\frac{x^{2}}{2}} \quad \text { and } \quad G(x)=\int_{0}^{x} \sqrt{\frac{2}{\pi}} e^{-\frac{t^{2}}{2}} d t=2 \Phi(x)-1 .
$$

The $c(\lambda)$ in Equation (1) can be calculated as

$$
c(\lambda)=\frac{1}{E\{G(\lambda Y)\}},
$$

where $Y$ follows the standard half-normal distribution.

Applying the relation $2 \Phi(x)-1=\operatorname{erf}\left(\frac{x}{\sqrt{2}}\right)$, see Gradshteyn and Ryzhik (2000), where

$$
\operatorname{erf}(x)=\frac{2}{\sqrt{\pi}} \sum_{k=0}^{\infty} \frac{(-1)^{k} x^{2 k+1}}{k !(2 k+1)}
$$

concludes

$$
\begin{aligned}
E\{G(\lambda Y)\} & =\int_{0}^{\infty} \frac{2}{\sqrt{\pi}} e^{-\frac{y^{2}}{2}} \operatorname{erf}\left(\frac{\lambda y}{\sqrt{2}}\right) d y \\
& =\frac{2 \sqrt{2}}{\pi} \sum_{k=0}^{\infty} \frac{(-1)^{k}\left(\frac{\lambda}{\sqrt{2}}\right)^{2 k+1}}{k !(2 k+1)} \int_{0}^{\infty} e^{-\frac{y^{2}}{2}} y^{2 k+1} d y \\
& =\frac{2 \sqrt{2}}{\pi} \sum_{k=0}^{\infty} \frac{(-1)^{k}\left(\frac{\lambda}{\sqrt{2}}\right)^{2 k+1}}{k !(2 k+1)} 2^{k} \Gamma(k+1) \\
& =\frac{2}{\pi} \arctan (\lambda)
\end{aligned}
$$

where $\Gamma(k)=\int_{0}^{\infty} t^{k-1} e^{-t} d t$. Thus,

$$
c(\lambda)=\frac{\pi}{2 \arctan (\lambda)} .
$$


It follows from (1) that the pdf of the PHN distribution is given by

$$
f(x ; \lambda)=\frac{\sqrt{\frac{\pi}{2}}}{\arctan (\lambda)} e^{-\frac{x^{2}}{2}} \operatorname{erf}\left(\frac{\lambda x}{\sqrt{2}}\right)=\frac{\sqrt{\frac{\pi}{2}}}{\arctan (\lambda)} e^{-\frac{x^{2}}{2}}(2 \Phi(\lambda x)-1), \quad x>0,
$$

where $\lambda>0$ and $\Phi(\cdot)$ denotes the standard normal distribution function. In what comes after this, we will denote the PHN distribution as $P H N(\lambda)$, for simplicity.

\section{Proposition 1.}

(i) If $x \rightarrow \infty$ or $x \rightarrow 0$, then $f(x) \rightarrow 0$.

(ii) The half-normal distribution can be obtained from the perturbed halfnormal distribution as $\lambda \rightarrow \infty$.

(iii) If $\lambda \rightarrow 0$ then the PHN distribution converges to a Rayleigh distribution with scale parameter 1.

Proposition 2. The PHN density function (5) can be demonstrated as the form

$$
f(x ; \lambda)=4 c(\lambda) \phi(x)\left[\Phi(\lambda x)-\frac{1}{2}\right], \quad x>0,
$$

where $\phi(\cdot)$ and $\Phi(\cdot)$ are the standard normal density and distribution functions and $c(\lambda)$ is given in Equation (4).

Thus, according to (6), we can see that the density is the weighted difference of a truncated, Azzalini skew normal density (Flecher et al., 2009) and half-normal density.

Proposition 3. The location-scale extension of the PHN distribution, denoted by $\operatorname{PHN}(\xi, \eta, \lambda)$, can be calculated as

$$
\begin{aligned}
f(x ; \xi, \eta, \lambda) & =\frac{c(\lambda)}{\eta} g\left(\frac{x-\xi}{\eta}\right) G\left(\frac{\lambda(x-\xi)}{\eta}\right) \\
& =\frac{\sqrt{\frac{\pi}{2}}}{\eta \arctan (\lambda)} e^{-\frac{(x-\xi)^{2}}{2 \eta^{2}}} \operatorname{erf}\left(\frac{\lambda(x-\xi)}{\sqrt{2} \eta}\right) \\
& =\frac{\sqrt{\frac{\pi}{2}}}{\eta \arctan (\lambda)} e^{-\frac{(x-\xi)^{2}}{2 \eta^{2}}}\left\{2 \Phi\left(\frac{\lambda(x-\xi)}{\eta}\right)-1\right\}, \quad x>\xi,(7)
\end{aligned}
$$

J. Statist. Res. Iran 14 (2017): 219-246 
where $\lambda(>0), \eta(>0)$ and $\xi(\in \mathbb{R})$ are the shape, scale and location parameters, respectively.

\section{Cumulative Distribution Function}

In this section, we derive the cdf of the PHN distribution using the series expansion for the error function in Equation (3).

Theorem 1. The cumulative distribution function of the PHN distribution is given by

$$
F(x)=1-\frac{1}{\arctan (\lambda)} \sum_{k=0}^{\infty} \frac{(-1)^{k} \lambda^{2 k+1}}{k !(2 k+1)} \Gamma\left(k+1, \frac{x^{2}}{2}\right),
$$

where $\Gamma\left(k+1, \frac{y^{2}}{2}\right)$ is the incomplete gamma function defined as

$$
\Gamma\left(k+1, \frac{y^{2}}{2}\right)=\int_{\frac{y^{2}}{2}}^{\infty} t^{k} e^{-t} d t .
$$

Proof. Equation (5) gives

$$
\begin{aligned}
F(x) & =\int_{0}^{x} \frac{\sqrt{\frac{\pi}{2}}}{\arctan (\lambda)} e^{-\frac{t^{2}}{2}} \operatorname{erf}\left(\frac{\lambda t}{\sqrt{2}}\right) d t \\
& =\frac{\sqrt{2}}{\arctan (\lambda)} \sum_{k=0}^{\infty} \frac{(-1)^{k}\left(\frac{\lambda}{\sqrt{2}}\right)^{2 k+1}}{(2 k+1) k !} \int_{0}^{x} e^{-\frac{t^{2}}{2}} t^{2 k+1} d t .
\end{aligned}
$$

Using this fact that $\int_{0}^{x} e^{-\frac{t^{2}}{2}} t^{2 k+1} d t=2^{k}\left[\Gamma(k+1)-\Gamma\left(k+1, \frac{x^{2}}{2}\right)\right]$, we have

$$
\begin{aligned}
F(x) & =\frac{\sqrt{2}}{\arctan (\lambda)} \sum_{k=0}^{\infty} \frac{(-1)^{k}\left(\frac{\lambda}{\sqrt{2}}\right)^{2 k+1}}{(2 k+1) k !}\left[2^{k}\left(k !-\Gamma\left(k+1, \frac{x^{2}}{2}\right)\right)\right] \\
& =\frac{\sqrt{2}}{\arctan (\lambda)}\left[\frac{1}{\sqrt{2}} \arctan (\lambda)-\frac{1}{\sqrt{2}} \sum_{k=0}^{\infty} \frac{(-1)^{k} \lambda^{2 k+1}}{(2 k+1) k !} \Gamma\left(k+1, \frac{x^{2}}{2}\right)\right] \\
& =1-\frac{1}{\arctan (\lambda)} \sum_{k=0}^{\infty} \frac{(-1)^{k} \lambda^{2 k+1}}{(2 k+1) k !} \Gamma\left(k+1, \frac{x^{2}}{2}\right) .
\end{aligned}
$$


It is easy to check that $\lim _{x \rightarrow \infty} F(x)=1$ and $\lim _{x \rightarrow 0} F(x)=0$. Figure 1 shows the graphs of pdf and cdf of the PHN distribution for different values of $\lambda$.
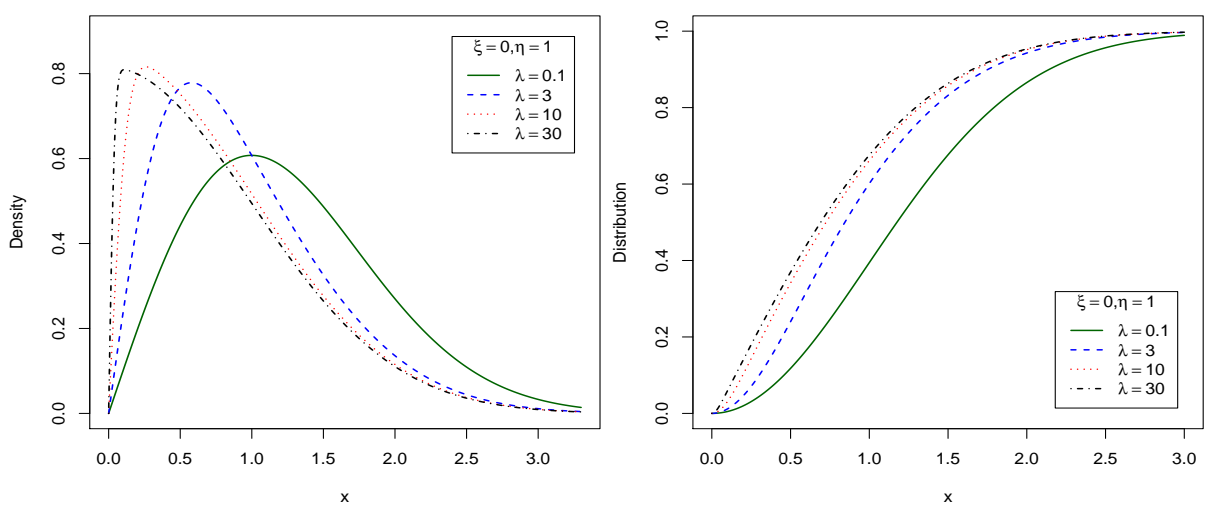

Figure 1. Graphs of pdf and cdf of the PHN distribution for different values of $\lambda$.

\subsection{Hazard Rate Function and Survival Function}

The survival function and hazard rate function defined by $s(x)=1-F(x)$ and $h(x)=\frac{f(x)}{s(x)}$, respectively are the two important quantity characterizing life phenomena. For the PHN distribution, $s(x)$ and $h(x)$ take the forms

$$
s(x)=\frac{1}{\arctan (\lambda)} \sum_{k=0}^{\infty} \frac{(-1)^{k} \lambda^{2 k+1}}{(2 k+1) k !} \Gamma\left(k+1, \frac{x^{2}}{2}\right)
$$

and

$$
h(x)=\frac{\sqrt{\frac{\pi}{2}} e^{\frac{-x^{2}}{2}}(2 \Phi(\lambda x)-1)}{\sum_{k=0}^{\infty} \frac{(-1)^{k} \lambda^{2 k+1}}{k !(2 k+1)} \Gamma\left(k+1, \frac{x^{2}}{2}\right)},
$$

respectively. The graphs of survival function and hazard rate function of the PHN distribution for different values $\lambda$ are shown in Figure 2.

Theorem 2. The hazard rate function of the PHN distribution is increasing (IFR). 

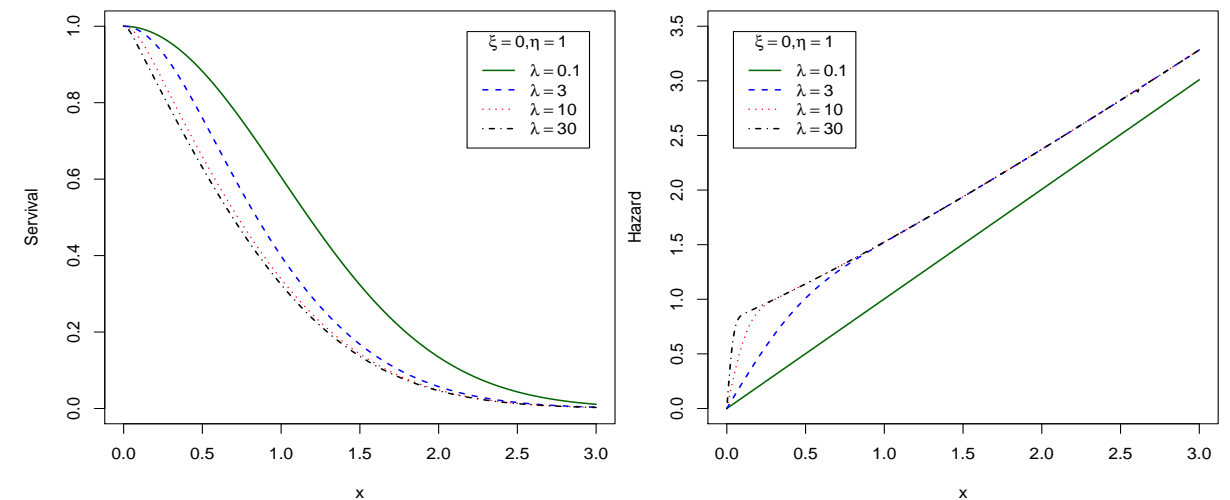

Figure 2. Graphs of survival function and hazard rate function of the PHN distribution for different values of $\lambda$.

Proof. This theorem is proved by following closely a theorem given by Glaser (1980). Define the function

$$
\eta(x)=f^{\prime}(x ; \lambda) / f(x ; \lambda)=-\frac{\partial}{\partial x} \log f(x ; \lambda),
$$

where $f^{\prime}(x ; \lambda)$ denotes the first derivative of $f(x ; \lambda)$ in $(5)$. To get properties of hazard rate function, it suffices to investigate on $\eta^{\prime}(x)=\frac{\partial^{2}}{\partial x^{2}} \log f(x ; \lambda)$. We have

$$
\eta(x)=x-\frac{2 \lambda \phi(\lambda x)}{2 \Phi(\lambda x)-1}
$$

and

$$
\eta^{\prime}(x)=1-\frac{2 \lambda^{2} \phi^{\prime}(\lambda x)(2 \Phi(\lambda x)-1)-4 \lambda^{2} \phi^{2}(\lambda x)}{(2 \Phi(\lambda x)-1)^{2}},
$$

where

$$
\phi^{\prime}(\lambda x)=-\frac{e^{-\frac{x^{2} \lambda^{2}}{2}} x \lambda^{2}}{\sqrt{2 \pi}} .
$$

Since the denominator of $\eta^{\prime}(x)$ is always positive, we must only investigate the expression of the numerator. Since $x>0$ and $\lambda>0,2 \Phi(\lambda x)-1$ is positive and so the first expression of the numerator is negative. Therefore, the expression of the numerator is negative and hence, $\eta^{\prime}(x)$ is positive. Thus from Glaser (1980), we conclude that the hazard rate function of the PHN 
distribution is increasing (IFR).

\section{Moments}

Moments play a basic role in finding the important properties of distributions such as skewness, kurtosis, asymmetric and etc. In this section, we derive the $r$ th moment about zero and the $r$ th central moment of the PHN distribution. First consider the well-known hypergeometric function (Gradshtryn and Ryzhik, (2000) defined by (for $\alpha_{k}>0, \beta_{k}>0, k=1,2, \ldots$ )

$$
{ }_{p} F_{q}\left(\alpha_{1}, \ldots, \alpha_{p} ; \beta_{1}, \ldots, \beta_{q} ; x\right)=\sum_{k=0}^{\infty} \frac{\left(\alpha_{1}\right)_{k} \ldots\left(\alpha_{p}\right)_{k}}{\left(\beta_{1}\right)_{k} \ldots\left(\beta_{q}\right)_{k}} \frac{x^{k}}{k !},
$$

where $(\alpha)_{i}=\alpha(\alpha+1) \ldots(\alpha+i-1)$ is the ascending factorial. An important particular case corresponds to $p=2$ and $q=1$ and leads to

$$
{ }_{2} F_{1}(a, b ; c ; x)=\sum_{k=0}^{\infty} \frac{(a)_{k}(b)_{k}}{(c)_{k}} \frac{x^{k}}{k !}
$$

Theorem 3. If the random variable $X$ has the PHN distribution then

$$
\mu_{r}^{\prime}=E\left(X^{r}\right)=\frac{2^{\frac{r}{2}} \lambda}{\arctan (\lambda)} \Gamma\left(\frac{r}{2}+1\right)_{2} F_{1}\left(\frac{1}{2}, 1+\frac{r}{2} ; \frac{3}{2} ;-\lambda^{2}\right) .
$$

Proof. According to Equation (5) and using the series expansion (3), the $r$ th moment about zero of the random variable $X$ with PHN distribution is given by

$$
\begin{aligned}
E\left(X^{r}\right) & =\frac{\sqrt{\frac{\pi}{2}}}{\arctan (\lambda)} \int_{0}^{\infty} x^{r} e^{-\frac{x^{2}}{2}} \operatorname{erf}\left(\frac{\lambda x}{\sqrt{2}}\right) d x \\
& =\frac{\sqrt{\frac{\pi}{2}}}{\arctan (\lambda)} \int_{0}^{\infty} x^{r} e^{-\frac{x^{2}}{2}} \frac{2}{\sqrt{\pi}} \sum_{k=0}^{\infty} \frac{(-1)^{k}\left(\frac{\lambda x}{\sqrt{2}}\right)^{2 k+1}}{(2 k+1) k !} d x \\
& =\frac{\sqrt{2}}{\arctan (\lambda)} \sum_{k=0}^{\infty} \frac{(-1)^{k}\left(\frac{\lambda}{\sqrt{2}}\right)^{2 k+1}}{(2 k+1) k !} \int_{0}^{\infty} x^{r+2 k+1} e^{-\frac{x^{2}}{2}} d x
\end{aligned}
$$




$$
\begin{aligned}
& =\frac{\sqrt{2}}{\arctan (\lambda)} \sum_{k=0}^{\infty} \frac{(-1)^{k}\left(\frac{\lambda}{\sqrt{2}}\right)^{2 k+1}}{(2 k+1) k !} 2^{k+\frac{r}{2}} \Gamma\left(k+\frac{r}{2}+1\right) \\
& =\frac{2^{\frac{r}{2}} \lambda}{\arctan (\lambda)} \Gamma\left(\frac{r}{2}+1\right)_{2} F_{1}\left(\frac{1}{2}, 1+\frac{r}{2} ; \frac{3}{2} ;-\lambda^{2}\right) .
\end{aligned}
$$

The following corollary provides explicit expressions for the first four order moments of the PHN distribution.

Corollary 1. The first four order moments of the PHN distribution are given by

$$
\begin{aligned}
\mu_{1}^{\prime} & =\frac{\lambda \sqrt{\pi}}{\arctan (\lambda) \sqrt{2 \lambda^{2}+2}}, \\
\mu_{2}^{\prime} & =1+\frac{\lambda}{\arctan (\lambda)\left(1+\lambda^{2}\right)}, \\
\mu_{3}^{\prime} & =\frac{\lambda\left(3+2 \lambda^{2}\right) \sqrt{\frac{\pi}{2}}}{\arctan (\lambda)\left(1+\lambda^{2}\right)^{\frac{3}{2}}}, \\
\mu_{4}^{\prime} & =3+\frac{\lambda\left(5+3 \lambda^{2}\right)}{\left(1+\lambda^{2}\right)^{2} \arctan (\lambda)} .
\end{aligned}
$$

According to the density function of the PHN distribution in (6), another method to derive the moments of the PHN distribution can be obtained by combining well-known results for the moments of the HN distribution with the results for the moments of the truncated skew-normal distribution (Flecher et al., 2009). Hence we have the following theorem.

Theorem 4. According to Proposition 2, another method to derive the rth moment of the PHN distribution can be obtained by

$\mu_{r}^{\prime}=E\left(X^{r}\right)=c(\lambda)\left[2 E\left(T^{r} \mid 0<T<\infty\right)-\sqrt{\frac{2}{\pi}} 2^{\frac{r-1}{2}} \Gamma\left(\frac{r+1}{2}\right)\right], \quad r=1,2, \ldots$, where $s_{\lambda, r}=E\left(T^{r} \mid 0<T<\infty\right)$ denotes the rth moment of the truncated skew-normal distribution. In above equation the following recursive relationship holds

$$
s_{\lambda, r}=(r-1) s_{\lambda, r-2}+\tau_{\lambda, r}, \quad \text { for } r=1,2, \ldots,
$$


where $s_{\lambda,-1}$ can be any finite value, $\lambda_{*}=\sqrt{\lambda^{2}+1}$,

$$
\tau_{\lambda, r}=-\frac{2 \int_{0}^{\infty} t^{r-1} \phi(t) \Phi(\lambda t) d t}{\left[F_{\lambda}(t)\right]_{0}^{\infty}}+\frac{1}{\sqrt{2 \pi}} \cdot \frac{\lambda}{\lambda_{*}^{r}\left[F_{\lambda}(t)\right]_{0}^{\infty} m_{r-1},}
$$

where $s_{\lambda, 0}=1, m_{-1}$ can be any finite value, $m_{0}=1, m_{r}=(r-1) m_{r-2}-$ $\sqrt{\frac{2}{\pi}} 2^{\frac{r-2}{2}} \Gamma\left(\frac{r}{2}\right)$, for $r=1,2, \ldots$, and $F_{\lambda}(t)$ denotes the cdf of the standard skew-normal distribution.

Proof. See Flecher et al. (2009) for more details in moments of truncated skew-normal variables.

Applying the expression $\mu_{r}=E\left(X-\mu_{1}^{\prime}\right)^{r}=\sum_{j=0}^{r}(-1)^{r-j}\left(\begin{array}{c}r \\ j\end{array}\right) \mu_{j}^{\prime}\left(\mu_{1}^{\prime}\right)^{r-j}$, the $r$ th central moment of the PHN distribution can be calculated as

$$
\begin{aligned}
\mu_{r} & =\sum_{j=0}^{r}(-1)^{r-j}\left(\begin{array}{l}
r \\
j
\end{array}\right) \mu_{j}^{\prime}\left(\mu_{1}^{\prime}\right)^{r-j} \\
& =\sum_{j=0}^{r}(-1)^{r-j}\left(\begin{array}{l}
r \\
j
\end{array}\right) \frac{\lambda 2^{\frac{j}{2}}}{\arctan (\lambda)} \sum_{k=0}^{\infty} \frac{(-1)^{k} \lambda^{2 k}}{k !(2 k+1)} \Gamma\left(k+\frac{j}{2}+1\right)\left(\mu_{1}^{\prime}\right)^{r-j} \\
& =\frac{\lambda}{\arctan (\lambda)} \sum_{j=0}^{\infty}\left(\begin{array}{l}
r \\
j
\end{array}\right)\left(\mu_{1}^{\prime}\right)^{r-j} 2^{\frac{j}{2}} \Gamma\left(\frac{j}{2}+1\right)_{2} F_{1}\left(\frac{1}{2}, \frac{j}{2}+1 ; \frac{3}{2} ; \lambda^{2}\right),
\end{aligned}
$$

where $\mu_{1}^{\prime}$ denotes the mean of the PHN distribution.

Other important indices of the shape of the distribution are the skewness, $\gamma_{1}=\frac{\mu_{3}}{\sigma^{3}}$ and kurtosis, $\gamma_{2}=\frac{\mu_{4}}{\sigma^{4}}$, where $\mu_{3}$ and $\mu_{4}$ are the third and fourth central moments about the mean, respectively and $\sigma$ denotes the standard deviation. For the PHN distribution these indices are given by

$$
\gamma_{1}=\frac{3.54491 \lambda^{2}\left(\pi \lambda-3 \arctan (\lambda)-\lambda[\arctan (\lambda)]^{2}\right)}{\left[-\pi \lambda^{2}+2 \arctan (\lambda)\left(\lambda+\left(1+\lambda^{2}\right) \arctan (\lambda)\right)\right]^{1.5}}
$$

and 
Table 1. Expectation, variance, skewness and kurtosis of the PHN distribution for different values of $\lambda$.

\begin{tabular}{ccccccc}
\hline \hline$\lambda$ & 0.1 & 0.5 & 1 & 3 & 10 & 100 \\
\hline $\mathrm{E}(\mathrm{X})$ & 1.25124 & 1.20889 & 1.12838 & 0.951925 & 0.847713 & 0.802956 \\
$\operatorname{Var}(\mathrm{X})$ & 0.427789 & 0.401308 & 0.36338 & 0.334022 & 0.348685 & 0.361668 \\
$\gamma_{1}$ & 0.63113 & 0.640333 & 0.703763 & 0.945647 & 1.00966 & 0.998085 \\
$\gamma_{2}$ & 3.24515 & 3.27077 & 3.43459 & 3.91037 & 3.93563 & 3.8776 \\
\hline
\end{tabular}

$$
\begin{aligned}
\gamma_{2}= & \left\{-3 \pi^{2} \lambda^{4}+4 \arctan (\lambda)\left(3 \pi \lambda^{3}-\pi \lambda^{2}\left(3+\lambda^{2}\right)\right.\right. \\
& \left.\left.\times \arctan (\lambda)+\lambda\left(5+3 \lambda^{2}\right) \arctan (\lambda)^{2}+3\left(1+\lambda^{2}\right)^{2} \arctan (\lambda)^{3}\right)\right\} \\
& /\left\{\pi \lambda^{2}-2 \arctan (\lambda)\left(\lambda+\left(1+\lambda^{2}\right) \arctan (\lambda)\right)\right\}^{2} .
\end{aligned}
$$

Table 1 gives the values of $E(X), \operatorname{Var}(X), \gamma_{1}$ and $\gamma_{2}$ of the PHN distribution for different values of $\lambda$.

The behavior of the expectation, variance, skewness and kurtosis of the PHN distribution, as a function of $\lambda$ is illustrated in Figure 3. Numerical calculations with MATHEMATICA, shows the following facts about the PHN distribution.

(i) $E(X)$ is decreasing for all values $\lambda>0$.

(ii) $\operatorname{Var}(X)$ is decreasing for $\lambda<2.6981$ and increasing for $\lambda>2.6981$.

(iii) $\gamma_{1}$ is increasing for $\lambda<10.228$ and decreasing for $\lambda>10.228$.

(iv) $\gamma_{2}$ is increasing for $\lambda<5.2526$ and decreasing for $\lambda>5.2526$.

\subsection{Moment Generating Function and Characteristic Func- tion}

Here we derive the moment generating function (mgf) and characteristic function of the PHN distribution. The moment generating function is given by 

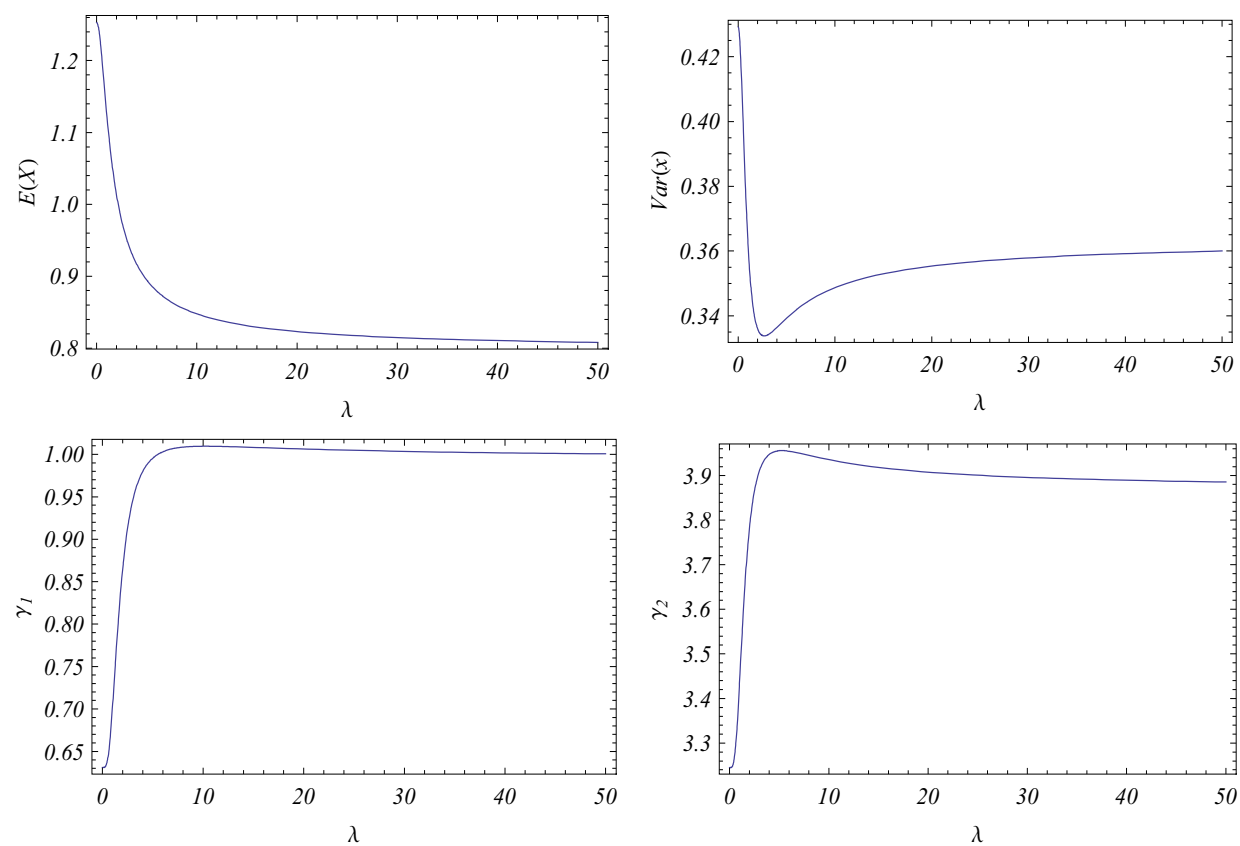

Figure 3. The plots of expectation, variance, skewness and kurtosis of the PHN distribution as a function of $\lambda$.

$$
\begin{aligned}
M_{X}(t) & =E\left(e^{t X}\right)=\sum_{j=0}^{\infty} \frac{t^{j}}{j !} E\left(X^{j}\right) \\
& =\sum_{j=0}^{\infty} \frac{t^{j}}{j !} \frac{\lambda 2^{\frac{j}{2}}}{\arctan (\lambda)} \sum_{k=0}^{\infty} \frac{(-1)^{k} \lambda^{2 k}}{k !(2 k+1)} \Gamma\left(k+\frac{j}{2}+1\right) \\
& =\frac{\lambda}{\arctan (\lambda)} \sum_{j=0}^{\infty} \frac{t^{j} 2^{\frac{j}{2}}}{j !} \Gamma\left(\frac{j}{2}+1\right)_{2} F_{1}\left(\frac{1}{2}, \frac{j}{2}+1, \frac{3}{2},-\lambda^{2}\right) .
\end{aligned}
$$

The characteristic function of the PHN distribution takes the form

$$
\phi(t)=\frac{\lambda}{\arctan (\lambda)} \sum_{j=0}^{\infty} \frac{(i t)^{j} 2^{\frac{j}{2}}}{j !} \Gamma\left(\frac{j}{2}+1\right)_{2} F_{1}\left(\frac{1}{2}, \frac{j}{2}+1, \frac{3}{2},-\lambda^{2}\right),
$$


Table 2. Values of mode of the PHN distribution for various values of $\lambda$.

\begin{tabular}{ccccccc}
\hline$\lambda$ & 0.1 & 0.5 & 1 & 3 & 10 & 100 \\
\hline mode & 0.99834 & 0.96189 & 0.87690 & 0.57856 & 0.26176 & 0.03904 \\
\hline
\end{tabular}

where $i=\sqrt{-1}$ is the complex imaginary unit.

\section{Other Properties of the PHN Distribution}

In this section, some important properties of the PHN distribution are discussed.

Theorem 5. The PHN distribution is strongly unimodal.

Proof. Since $f(x ; \lambda)$ vanishes as $x \rightarrow 0$ or $x \rightarrow \infty$, it is enough to show that the pdf of the PHN distribution is log-concave. Therefor it's enough to show that $\frac{d^{2}}{d x^{2}} \log f(x ; \lambda)<0$, where $f(x ; \lambda)$ is given in $(5)$. The first and second derivative of $\log f(x ; \lambda)$ with respect to $\lambda$ are given, respectively by

$$
\frac{d}{d x} \log f(x)=-x+\frac{\sqrt{\frac{2}{\pi}} \lambda e^{-\frac{\lambda^{2} x^{2}}{2}}}{\operatorname{erf}\left(\frac{\lambda x}{\sqrt{2}}\right)},
$$

and

$$
\frac{d^{2}}{d x^{2}} \log f(x)=-1-\frac{2 \lambda^{2} e^{-\lambda^{2} x^{2}}}{\pi \operatorname{erf}\left(\frac{\lambda x}{\sqrt{2}}\right)}-\frac{\sqrt{\frac{2}{\pi}} x \lambda^{3} e^{-\frac{\lambda^{2} x^{2}}{2}}}{\operatorname{erf}\left(\frac{\lambda x}{\sqrt{2}}\right)} .
$$

Since $\frac{d^{2}}{d x^{2}} \log f(x)<0$, thus the pdf of the PHN distribution is always unimodal.

According to Theorem 5, the PHN distribution has a single mode at $x=x_{0}$, where $x_{0}$ is the solution to the equation obtained by equating the first derivative (10) to 0 .

Table 2 gives values of the mode of the PHN distribution for different values of $\lambda$.

The following theorem gives a method for generating a random sample from the PHN distribution.

Theorem 6. Suppose that $X_{1}$ and $X_{2}$ are two independent and identically distributed (i.i.d.) random variables with the pdf $g_{Y}(\cdot)$ and $c d f G_{Y}(\cdot)$ in (2), 
then for any $\lambda>0$, a new random variable $X=X_{1}$ given that $\lambda X_{1}>X_{2}$ has the PHN distribution.

Proof. Consider the random variable $X=X_{1} \mid\left\{\lambda X_{1}>X_{2}\right\}$. We have

$$
\begin{aligned}
F(x)= & P\left(X_{1} \leqslant x \mid \lambda X_{1}>X_{2}\right)=\frac{P\left(X_{1} \leqslant x, \lambda X_{1}>X_{2}\right)}{\int_{0}^{\infty} \int_{0}^{\lambda x_{1}} g_{Y}\left(x_{2}\right) g_{Y}\left(x_{1}\right) d x_{2} d x_{1}} \\
& =\frac{\int_{0}^{x} P\left(X_{1}=x_{1}, X_{2}<\lambda X_{1}\right) d x_{1}}{\frac{1}{c(\lambda)}}=c(\lambda) \int_{0}^{x} g\left(x_{1}\right) G\left(\lambda x_{1}\right) d x_{1} .
\end{aligned}
$$

Therefore $f(x)=c(\lambda) g(x) G(\lambda x)$, where $c(\lambda)$ is given in (4). So

$$
X=X_{1} \mid\left\{\lambda X_{1}>X_{2}\right\} \sim P H N(\lambda) .
$$

\section{Mean Deviation about the Mean}

The amount of scatter in a population can be expressed as the mean deviation about the mean, which is defined by

$$
\delta_{1}(X)=\int_{0}^{\infty}|x-\mu| f(x) d x=2 \mu F(\mu)-2 I(\mu),
$$

where $\mu=E(X)$ and $I(\mu)=\int_{0}^{\mu} x f(x) d x$. We have

$$
\begin{aligned}
I(\mu) & =\frac{-\sqrt{\frac{\pi}{2}}\left\{e^{\frac{-\mu^{2}}{2}}(2 \Phi(\mu \lambda)-1)-\frac{\lambda\left(2 \Phi\left(\mu \sqrt{1+\lambda^{2}}\right)-1\right)}{\sqrt{1+\lambda^{2}}}\right\}}{\arctan (\lambda)} \\
& =\frac{\sqrt{\frac{\pi}{2}} \lambda\left(2 \Phi\left(\mu \sqrt{1+\lambda^{2}}\right)-1\right)}{\arctan (\lambda) \sqrt{1+\lambda^{2}}}-f(\mu),
\end{aligned}
$$


Hence, the measure $\delta_{1}(X)$ can be calculated as

$$
\delta_{1}(X)=2 \mu F(\mu)+2 f(\mu)-\frac{\sqrt{2 \pi} \lambda\left(2 \Phi\left(\mu \sqrt{1+\lambda^{2}}\right)-1\right)}{\arctan (\lambda) \sqrt{1+\lambda^{2}}},
$$

where $F(\cdot)$ and $f(\cdot)$ are given in (5) and (8).

\section{Estimation}

Here, we consider estimation of the parameters of the PHN distribution by the method of moments and maximum likelihood and provide expressions for the associated Fisher information matrix.

\subsection{Method of Moments}

Suppose that $x_{1}, \ldots, x_{n}$ is an observed random sample from the locationscale PHN distribution with pdf (7). For the moments estimation, let $m_{1}=$ $(1 / n) \sum_{j=1}^{n} x_{j}, m_{2}=(1 / n) \sum_{j=1}^{n} x_{j}^{2}$ and $m_{3}=(1 / n) \sum_{j=1}^{n} x_{j}^{3}$ denote the first, second and third sample moments. Equating three first moments of (7) with the sample moments $m_{1}, m_{2}$ and $m_{3}$, gives the equations

$$
\begin{aligned}
& \frac{\eta \lambda \sqrt{\pi}}{\arctan (\lambda) \sqrt{2 \lambda^{2}+2}}+\xi=m_{1} \\
& \eta^{2}\left[1+\frac{\lambda}{\arctan (\lambda)\left(\lambda^{2}+1\right)}\right]+2 \xi m_{1}-\xi^{2}=m_{2},
\end{aligned}
$$

and

$$
\eta^{3}\left[\frac{\lambda\left(3+2 \lambda^{2}\right) \sqrt{\pi / 2}}{\arctan (\lambda)\left(\lambda^{2}+1\right)^{3 / 2}}\right]+3 m_{2} \xi-3 m_{1} \xi^{2}+\xi^{3}=m_{3} .
$$

These equations cannot be solved analytically and statistical software can be used to obtain the MMEs numerically. A simulation method based on $\mathrm{R}$ software is used in order to obtain the MMEs of the parameters $\xi, \eta$ and $\lambda$ in Section 8.

\subsection{Method of Maximum Likelihood}

In this part, estimation of the parameters of the PHN distribution, by the method of maximum likelihood, is considered. If $Y$ follows the PHN distribution (7) with vector of parameters $\Theta=(\xi, \eta, \lambda)^{T}$, then the log-likelihood 
for the model parameters from a single observation $y$ of $Y$ is given by

$$
\begin{aligned}
l(\Theta)= & \log \left(\sqrt{\frac{\pi}{2}}\right)-\log \{\arctan (\lambda)\}-\log (\eta)-\frac{(y-\xi)^{2}}{2 \eta^{2}} \\
& +\log \left\{G\left(\frac{\lambda(y-\xi)}{\eta}\right)\right\}, \quad \xi<y,
\end{aligned}
$$

where $G(\cdot)$ denotes the cdf of the standard half-normal distribution in Equation (2). For an observed random sample $y=\left(y_{1}, \ldots, y_{n}\right)^{T}$ of size $n$ from $Y$, the maximum likelihood estimators (MLEs) of $\Theta$ can be obtained by maximizing the total log-likelihood function

$$
\begin{aligned}
l_{n}(\Theta \mid y)= & \frac{n}{2} \log \left(\frac{\pi}{2}\right)-n \log \{\arctan (\lambda)\}-n \log (\eta)-\sum_{i=1}^{n} \frac{\left(y_{i}-\xi\right)^{2}}{2 \eta^{2}} \\
& +\sum_{i=1}^{n} \log \left\{G\left(\frac{\lambda\left(y_{i}-\xi\right)}{\eta}\right)\right\} .
\end{aligned}
$$

For given $\xi$, the MLEs of $\eta$ and $\lambda, \hat{\eta}(\xi)$ and $\hat{\lambda}(\xi)$ respectively, can be achieved as

$$
\hat{\eta}(\xi)=\frac{\partial l_{n}}{\partial \eta}=-\frac{n}{\eta}+\sum_{i=1}^{n} \frac{\left(y_{i}-\xi\right)^{2}}{\eta^{3}}-\sum_{i=1}^{n} \frac{\lambda\left(y_{i}-\xi\right)}{\eta^{2}} w\left(y_{i}\right),
$$

and

$$
\hat{\lambda}(\xi)=\frac{\partial l_{n}}{\partial \lambda}=\frac{-n}{\left(1+\lambda^{2}\right) \arctan (\lambda)}+\sum_{i=1}^{n} w\left(y_{i}\right)\left(\frac{y_{i}-\xi}{\eta}\right),
$$

where $w\left(y_{i}\right)=\frac{g\left(\frac{\lambda\left(y_{i}-\xi\right)}{\eta}\right)}{G\left(\frac{\lambda\left(y_{i}-\xi\right)}{\eta}\right)}$. The MLE of $\xi$ can be reached by maximizing the profile log-likelihood function $l_{n}(\xi, \hat{\eta}(\xi), \hat{\lambda}(\xi) \mid y)$ with respect to $\xi$.

The three-parameter PHN distribution doesn't satisfy the regularity conditions of maximum likelihood estimation because the support of the distribution depends on the location parameter $\xi$. For the case where $\xi$ is known (for example $\xi=0)$, then $\sqrt{n}(\widehat{\eta}-\eta, \widehat{\lambda}-\lambda)$ is asymptotically $N_{2}\left(\mathbf{0}, I_{n}{ }^{-1}\right)$, where

$$
I_{n}=-\frac{1}{n}\left[\begin{array}{cc}
E\left(\frac{\partial^{2} l_{n}}{\partial \eta^{2}}\right) & E\left(\frac{\partial^{2} l_{n}}{\partial \eta \partial \lambda}\right) \\
E\left(\frac{\partial^{2} l_{n}}{\partial \lambda \partial \eta}\right) & E\left(\frac{\partial^{2} l_{n}}{\partial \lambda^{2}}\right)
\end{array}\right]
$$


with the second-order derivatives of $l_{n}(\Theta \mid y)$ with respect to $\eta$ and $\lambda$

$$
\begin{aligned}
& \frac{\partial^{2} l_{n}}{\partial \eta^{2}}=\frac{1}{\eta^{2}}\left\{n-3 \sum_{i=1}^{n} t_{i}^{2}+2 \lambda \sum_{i=1}^{n} t_{i} w\left(t_{i}\right)-\lambda^{3} \sum_{i=1}^{n} t_{i}^{3} w\left(t_{i}\right)-\lambda^{2} \sum_{i=1}^{n} t_{i}^{2} w^{2}\left(t_{i}\right)\right\}, \\
& \frac{\partial^{2} l_{n}}{\partial \lambda^{2}}=\frac{n+2 n \lambda \arctan (\lambda)}{\left[\left(1+\lambda^{2}\right) \arctan (\lambda)\right]^{2}}-\lambda \sum_{i=1}^{n} t_{i}^{3} w\left(t_{i}\right)-\sum_{i=1}^{n} t_{i}^{2} w^{2}\left(t_{i}\right),
\end{aligned}
$$

and

$$
\frac{\partial^{2} l_{n}}{\partial \eta \partial \lambda}=\frac{1}{\eta}\left\{-\sum_{i=1}^{n} t_{i} w\left(t_{i}\right)+\lambda^{2} \sum_{i=1}^{n} t_{i}^{3} w\left(t_{i}\right)+\lambda \sum_{i=1}^{n} t_{i}^{2} w^{2}\left(t_{i}\right)\right\} .
$$

This asymptotic behaviour remains valid if $I_{n}$ is replaced by the average sample information matrix, say $\widehat{I}_{n}$. The estimated asymptotic multivariate normal $N_{2}\left((\eta, \lambda)^{T}, \widehat{I}_{n}^{-1}\right)$ distribution can be used to construct approximate confidence intervals and hypothesis test for the parameters. The $100(1-\gamma)$ asymptotic confidence intervals for the parameters $\eta$ and $\lambda$ are given by

$$
\left(\widehat{\eta}-Z_{\frac{\gamma}{2}} \sqrt{\widehat{I}_{n}^{11}}, \widehat{\eta}+Z_{\frac{\gamma}{2}} \sqrt{\widehat{I}_{n}^{11}}\right) \quad \text { and } \quad\left(\widehat{\lambda}-Z_{\frac{\gamma}{2}} \sqrt{{\widehat{I_{n}}}^{22}}, \widehat{\lambda}+Z_{\frac{\gamma}{2}} \sqrt{{\widehat{I_{n}}}^{22}}\right)
$$

where ${\widehat{I_{n}}}^{11}$ and $\widehat{I}_{n}^{22}$ are the $(1,1)$ and $(2,2)$ diagonal elements of $\widehat{I}_{n}^{-1}$ and $Z_{\frac{\gamma}{2}}$ is the $(1-\gamma / 2)$ th quantile of the standard normal distribution.

Now, we will discuss the asymptotic properties of the MLEs for the nonregular case. By (7), when $\xi \neq 0$, the support of $\operatorname{PHN}(\xi, \eta, \lambda)$ depends on the unknown parameter $\xi$. For an observed random sample $y_{1}, \ldots, y_{n}$ from (7), the MLEs of $\Theta$ can be obtained by maximizing the total log-likelihood function

$$
\begin{aligned}
l_{n}(\Theta)= & \frac{n}{2} \log \left(\frac{\pi}{2}\right)-n \log \{\arctan (\lambda)\}-n \log (\eta)-\sum_{i=1}^{n} \frac{\left(y_{(i)}-\xi\right)^{2}}{2 \eta^{2}} \\
& +\sum_{i=1}^{n} \log \left\{G\left(\frac{\lambda\left(y_{(i)}-\xi\right)}{\eta}\right)\right\},
\end{aligned}
$$

where $y_{(1)}<y_{(2)}<\cdots<y_{(n)}$ denote the ordered $y_{i}$ 's. Instantly, from (14) it results that for fixed $\eta$ and $\lambda, l_{n}(\Theta) \rightarrow \infty$ as $\xi \uparrow y_{(1)}$. Thus, the MLEs do not exist in this case. 
Hence, to estimate the unknown parameters, the most natural way is to first estimate the location parameter $\xi$ by its consistent estimator $\hat{\xi}=y_{(1)}$, Smith (1985). Then, the modified log-likelihood function based on the remaining $(n-1)$ observations after ignoring the smallest observation and replacing $\xi$ by $\hat{\xi}$ is as following

$$
\begin{aligned}
l_{n}(\hat{\xi}, \eta, \lambda)= & \frac{n-1}{2} \log \left(\frac{\pi}{2}\right)-(n-1) \log \{\arctan (\lambda)\}-(n-1) \log (\eta) \\
& -\sum_{i=2}^{n} \frac{\left(y_{(i)}-y_{(1)}\right)^{2}}{2 \eta^{2}}+\sum_{i=2}^{n} \log \left\{G\left(\frac{\lambda\left(y_{(i)}-y_{(1)}\right)}{\eta}\right)\right\} .
\end{aligned}
$$

Now, by differentiating $l_{n}(\hat{\xi}, \eta, \lambda)$ with respect to $\eta$ and $\lambda$, respectively, and setting the results equal to zero, we have

$$
\frac{\partial l_{n}(\hat{\xi}, \eta, \lambda)}{\partial \eta}=-\frac{n-1}{\eta}+\sum_{i=2}^{n} \frac{\left(y_{(i)}-y_{(1)}\right)^{2}}{\eta^{3}}-\sum_{i=2}^{n} \frac{\lambda\left(y_{(i)}-y_{(1)}\right)}{\eta^{2}} w\left(y_{(i)}\right),
$$

and

$$
\frac{\partial l_{n}(\hat{\xi}, \eta, \lambda)}{\partial \lambda}=\frac{-(n-1)}{\left(1+\lambda^{2}\right) \arctan (\lambda)}+\sum_{i=2}^{n} w\left(y_{(i)}\right)\left(\frac{y_{(i)}-y_{(1)}}{\eta}\right),
$$

and the maximum likelihood estimates are obtained by solving iteratively the above equations.

\section{Simulation}

We carried out Monte Carlo simulations in order to compare the performance of all the estimators proposed in the preceding section. We used several different sample sizes and parameter values. We used the nlminb and optim functions of the $\mathrm{R}$ software to obtain the MMEs and MLEs of the parameters, respectively.

We considered five values for $n$, i.e., $n \in\{20,30,100,200,300\}$ and three values for the shape parameter $\lambda \in\{0.1,0.7,3\}$. In all cases, we set the location and scale parameters $\xi=0$ and $\eta=1$. For each values of model parameters and sample size, We compute the MMEs and MLEs of $\xi, \eta$ and $\lambda$. We simulated 1000 vectors of size $n$ of independent replicates and computed the average estimators (AE), the biases and the mean squared errors (MSEs). The following algorithm shows how a random sample from the PHN 
Table 3. Simulated average MMEs, mean squared errors and the bias of the parameters of the PHN distribution.

\begin{tabular}{|c|c|c|c|c|c|c|c|c|c|c|}
\hline \multirow[b]{2}{*}{$n$} & & $\xi=0$ & $\eta=1$ & $\lambda=0.1$ & $\xi=0$ & $\eta=1$ & $\lambda=0.7$ & $\xi=0$ & $\eta=1$ & $\lambda=3$ \\
\hline & & $\mathrm{AE}$ & MSE & Bias & $\mathrm{AE}$ & MSE & Bias & $\mathrm{AE}$ & MSE & Bias \\
\hline \multirow[t]{3}{*}{30} & $\xi$ & 0.065 & 0.032 & 0.072 & 0.004 & 0.002 & 0.004 & -0.047 & 0.018 & -0.055 \\
\hline & $\eta$ & 1.032 & 0.030 & 0.029 & 1.019 & 0.005 & 0.019 & 0.959 & 0.036 & -0.041 \\
\hline & $\lambda$ & 0.568 & 1.076 & 0.470 & 0.860 & 0.788 & 0.160 & 2.976 & 60.279 & -7.020 \\
\hline \multirow[t]{3}{*}{50} & $\xi$ & 0.048 & 0.018 & 0.056 & 0.003 & 0.002 & 0.003 & -0.041 & 0.012 & -0.042 \\
\hline & $\eta$ & 1.026 & 0.021 & 0.020 & 1.019 & 0.004 & 0.019 & 0.945 & 0.025 & -0.055 \\
\hline & $\lambda$ & 0.545 & 0.877 & 0.440 & 0.845 & 0.587 & 0.145 & 2.460 & 6.201 & -0.540 \\
\hline \multirow[t]{3}{*}{100} & $\xi$ & 0.034 & 0.008 & 0.044 & -0.006 & 0.001 & -0.006 & -0.037 & 0.008 & -0.030 \\
\hline & $\eta$ & 1.018 & 0.013 & 0.016 & 1.019 & 0.004 & 0.019 & 0.974 & 0.012 & -0.026 \\
\hline & $\lambda$ & 0.524 & 0.708 & 0.420 & 0.839 & 0.448 & 0.139 & 2.858 & 5.750 & -0.140 \\
\hline \multirow[t]{3}{*}{200} & $\xi$ & 0.021 & 0.004 & 0.011 & -0.025 & 0.002 & -0.025 & -0.025 & 0.005 & -0.024 \\
\hline & $\eta$ & 1.01 & 0.009 & 0.010 & 1.006 & 0.001 & 0.006 & 0.983 & 0.006 & -0.017 \\
\hline & $\lambda$ & 0.448 & 0.498 & 0.350 & 0.730 & 0.112 & 0.030 & 3.132 & 4.957 & 0.130 \\
\hline \multirow[t]{3}{*}{300} & $\xi$ & 0.015 & 0.002 & 0.007 & -0.034 & 0.002 & -0.034 & -0.021 & 0.004 & -0.017 \\
\hline & $\eta$ & 1.007 & 0.006 & 0.005 & 1.003 & 0.001 & 0.003 & 0.989 & 0.004 & -0.011 \\
\hline & $\lambda$ & 0.395 & 0.378 & 0.290 & 0.713 & 0.055 & 0.013 & 3.120 & 4.013 & 0.120 \\
\hline
\end{tabular}

distribution generate by Theorem 6, used in our simulation:

Step 1. For each value of sample $\left(x_{1}, \ldots, x_{n}\right)$, first it generates $w$ and $y$ from half-normal distribution.

Step 2. In next step, check the Theorem 6 condition, if $y<\lambda w$, then $x_{i}=$ $\xi+\eta w$.

Step 3. Finally, it returns a vector of $x_{i}$ with PHN distribution.

The results for the MMEs and MLEs are reported in Tables 3 and 4, respectively. 
Table 4. Simulated average MLEs, mean squared errors and the bias of the parameters of the PHN distribution.

\begin{tabular}{|c|c|c|c|c|c|c|c|c|c|c|}
\hline \multirow[b]{2}{*}{$n$} & & $\xi=0$ & $\eta=1$ & $\lambda=0.1$ & $\xi=0$ & $\eta=1$ & $\lambda=0.7$ & $\xi=0$ & $\eta=1$ & $\lambda=3$ \\
\hline & & $\mathrm{AE}$ & MSE & Bias & $\mathrm{AE}$ & MSE & Bias & $\mathrm{AE}$ & MSE & Bias \\
\hline \multirow[t]{3}{*}{30} & $\xi$ & 0.130 & 0.031 & 0.130 & 0.114 & 0.027 & 0.114 & 0.047 & 0.008 & 0.047 \\
\hline & $\eta$ & 1.013 & 0.026 & 0.013 & 0.994 & 0.025 & -0.006 & 0.940 & 0.026 & -0.060 \\
\hline & $\lambda$ & 2.733 & 28.912 & 2.633 & 3.259 & 28.260 & 2.559 & 4.937 & 22.257 & 1.937 \\
\hline \multirow[t]{3}{*}{50} & $\xi$ & 0.071 & 0.014 & 0.071 & 0.067 & 0.012 & 0.067 & -0.006 & 0.002 & -0.006 \\
\hline & $\eta$ & 1.028 & 0.015 & 0.028 & 1.020 & 0.019 & 0.020 & 0.968 & 0.017 & -0.032 \\
\hline & $\lambda$ & 1.394 & 9.009 & 1.294 & 2.008 & 9.408 & 1.308 & 4.868 & 10.128 & -0.132 \\
\hline \multirow[t]{3}{*}{100} & $\xi$ & 0.024 & 0.005 & 0.024 & 0.016 & 0.004 & 0.016 & -0.034 & 0.002 & -0.034 \\
\hline & $\eta$ & 1.036 & 0.010 & 0.036 & 1.019 & 0.012 & 0.019 & 0.983 & 0.009 & -0.017 \\
\hline & $\lambda$ & 0.667 & 1.672 & 0.567 & 1.008 & 1.662 & 0.308 & 3.814 & 5.108 & -1.186 \\
\hline \multirow[t]{3}{*}{200} & $\xi$ & -0.014 & 0.002 & -0.014 & -0.020 & 0.002 & -0.020 & -0.054 & 0.004 & -0.054 \\
\hline & $\eta$ & 1.028 & 0.005 & 0.028 & 1.004 & 0.006 & 0.004 & 0.992 & 0.005 & -0.008 \\
\hline & $\lambda$ & 0.300 & 0.338 & 0.200 & 0.561 & 0.495 & -0.139 & 3.140 & 4.799 & -1.860 \\
\hline \multirow[t]{3}{*}{300} & $\xi$ & -0.025 & 0.002 & -0.025 & -0.033 & 0.002 & -0.033 & -0.063 & 0.004 & -0.063 \\
\hline & $\eta$ & 1.028 & 0.003 & 0.028 & 1.002 & 0.004 & 0.002 & 1.001 & 0.003 & 0.001 \\
\hline & $\lambda$ & 0.208 & 0.158 & 0.108 & 0.427 & 0.392 & -0.273 & 3.054 & 4.541 & -1.946 \\
\hline
\end{tabular}

\section{Application}

To show the superiority of the PHN distribution, we compare the results of fitting the PHN distribution to some models such as log-normal (LN), Birnbaum-Saunders (BS), half-normal (HN), Weibull and gamma distributions (see Appendix), using two real data sets. The required numerical evaluations are implemented using the $\mathrm{R}$ software. The empirical scaled TTT transform (Aarset, 1987) can be used to identify the shape of the hazard rate function. The scaled TTT transform is convex (concave) if the hazard rate is decreasing (increasing), and for bathtub (unimodal) hazard rates, the scaled TTT transform is first convex (concave) and then concave (convex). 
The first data we consider is the uncensored data set from Nichols and Padgett (2006). The data gives 100 observations on tensile strength of carbon fibres. The TTT plot for this data in Figure 4 shows an increasing hazard rate function and, therefore, indicates the appropriateness of the PHN distribution to fit this data.

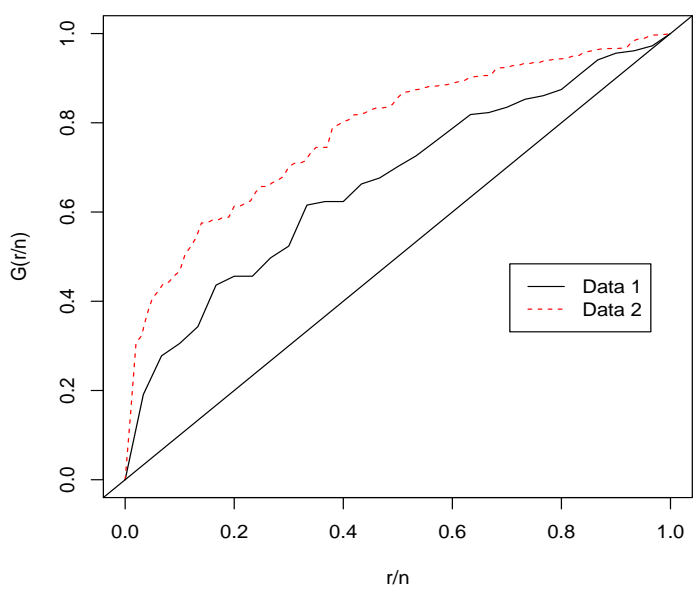

Figure 4. TTT-plots on Nichols and Padgett data (Data 1) and Hinkley data (Data 2).

Table 5 lists the MLEs of the parameters, AIC (Akaike Information Criterion), the Cramer-von Mises (CM) test, Watson (WA) test and the values of following K-S (Kolmogorov-Smirnov) statistic

$$
D_{n}=\sup _{x \in \mathbb{R}}\left|F_{n}(x)-F(x)\right|,
$$

where $F_{n}(x)$ is empirical distribution. In order to obtain its respective $p$ value, for small values of $n(\leqslant 40)$, the existing probability distribution of $D_{n}$ tables (Leslie and Miller, 1956) is used and for large values of $n$, the asymptotic distribution of $D_{n}$ is used as following

$$
p-\text { value }=P\left(D_{n} \geqslant z\right) \approx 2 P\left(\chi^{2}(2) \geqslant 4 n z^{2}\right) .
$$

Here, the ks.test functions of the $\mathrm{R}$ software is applied to get the values of Kolmogorov-Smirnov statistic and its corresponding $p$-value. The CM and WA test statistics are described in details in Chen (1999) and Watson 
Table 5. MLEs, AIC, K-S statistics, CM, WA and $p$-values for Nichols and Padgett data.

\begin{tabular}{llccccc}
\hline \hline Model & \multicolumn{1}{c}{ MLEs } & AIC & K-S & CM & WA & $p$-value \\
\hline PHN & $\widehat{\xi}=0.3361, \widehat{\eta}=1.7666$, & 294.96 & 0.093 & 0.3019 & 0.2827 & 0.35 \\
& $\widehat{\lambda}=0.0279$ & & & & & \\
LN & $\widehat{\mu}=0.8774, \widehat{\sigma}=0.4439$ & 300.84 & 0.118 & 0.3578 & 0.3117 & 0.13 \\
BS & $\widehat{\alpha}=0.4621, \widehat{\beta}=2.3660$ & 304.12 & 0.131 & 0.4399 & 0.3470 & 0.07 \\
Weibull & $\widehat{\alpha}=2.7929, \widehat{\sigma}=2.9437$ & 287.059 & 0.153 & 0.7581 & 0.2709 & 0.02 \\
HN & $\widehat{\mu}=0.3900, \widehat{\sigma}=2.2314$ & 330.12 & 0.273 & 2.9484 & 1.0557 & 0.00 \\
Gamma & $\widehat{k}=5.9526, \widehat{\theta}=0.8861$ & 524.05 & 0.661 & 18.1747 & 3.6456 & 0.00 \\
\hline
\end{tabular}

(1961), respectively. In general, the smaller values of CM and WA, indicate the better the fit to the data. From the values of these statistics, we conclude that the PHN distribution provides a better fit to this data than the LN, BS, Weibull, HN and gamma models. Although Weibull model provides AIC value smaller than PHN, its goodness of fit is rejected with $p$-value $=0.02$.

As a second application, we consider an uncensored data set from Hinkley (1977). This data consists of thirty successive values of March precipitation (in inches) in Minneapolis/St Paul. The TTT plot for this data in Figure 4 shows an increasing hazard rate function and indicates that appropriateness of the PHN distribution to fit this data. The MLEs of the parameters, the values of $\mathrm{K}$-S statistic with its respective $p$-value, AIC, CM and WA tests are listed in Table 6. From these values, we note that the PHN model is better than the LN, BS, Weibull and HN distributions and is a strong competitor to gamma distribution. Here, since the values of AIC are very close $(<2$ units), all the models are competitors and to compare their goodness of fit, the K-S, CM and WA statistics should be considered. The smallest values of these statistics show the best fit.

Plots of the estimated pdf and cdf of the PHN, LN, BS, HN, Weibull and gamma models fitted to the data sets corresponding to Tables 5 and 6 , respectively, are given in Figures 5 and 6.

\section{Conclusion}

We proposed the perturbed half-normal (PHN) distribution to extend the half-normal $(\mathrm{HN})$ distribution. We derived important properties of the new distribution and obtained closed-form expressions for its moments. Applica- 
Table 6. MLEs, AIC, K-S statistics, CM, WA and $p$-values for Hinkley data.

\begin{tabular}{llccccc}
\hline \hline Model & MLEs & AIC & K-S & CM & WA & $p$-value \\
\hline Gamma & $\widehat{k}=2.9582, \widehat{\theta}=0.5662$ & 80.20 & 0.056 & 0.0945 & 0.0943 & 1.00 \\
PHN & $\widehat{\xi}=0.0125, \widehat{\eta}=1.3662$, & 80.64 & 0.086 & 0.1049 & 0.1013 & 0.98 \\
& $\widehat{\lambda}=0.0345$ & & & & & \\
LN & $\widehat{\mu}=0.3374, \widehat{\sigma}=0.6226$ & 80.95 & 0.091 & 0.1116 & 0.1079 & 0.96 \\
BS & $\widehat{\alpha}=0.6502, \widehat{\beta}=1.3804$ & 80.75 & 0.105 & 0.1194 & 0.1108 & 0.90 \\
HN & $\widehat{\mu}=0.3200, \widehat{\sigma}=1.3550$ & 81.59 & 0.174 & 0.3220 & 0.1279 & 0.32 \\
Weibull & $\widehat{\alpha}=1.8089, \widehat{\sigma}=1.8923$ & 81.29 & 0.416 & 2.3556 & 0.4794 & 0.00 \\
\hline
\end{tabular}
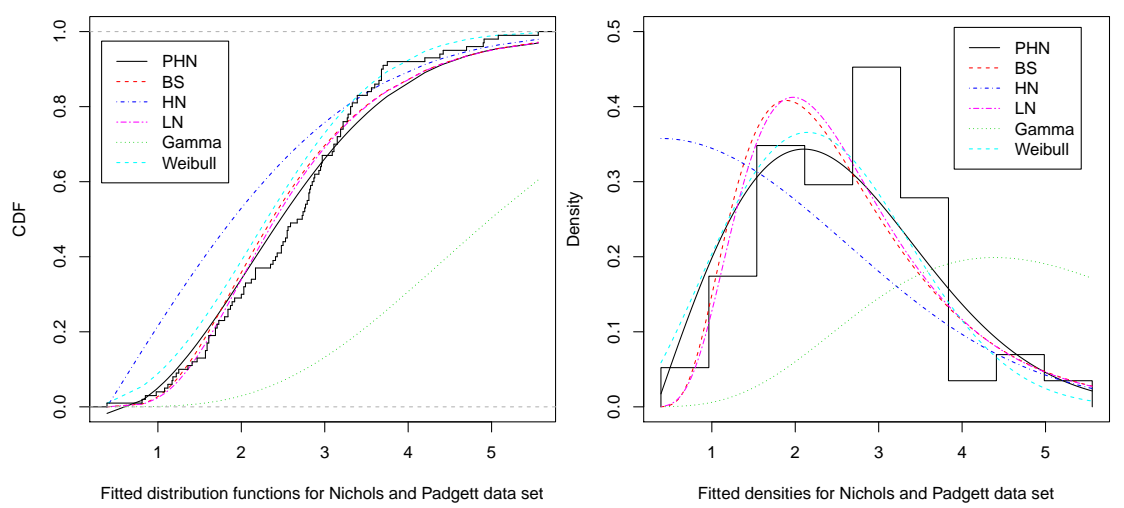

Figure 5. Left panel: Estimated distribution function from the fitted PHN, LN, BS, HN, Weibull and gamma distributions versus the empirical distribution for Nichols and Padgett data. Right panel: Estimated densities of the PHN, LN, BS, HN, Weibull and gamma models for Nichols and Padgett data.

tions of the PHN distribution to two real data sets are given to show that the new distribution provides consistently better fits than the log-normal (LN), Birnbaum-Saunders (BS), half-normal (HN), Weibull and gamma distributions. We hope that this generalization may attract wider applications in the lifetime analysis.

\section{Acknowledgement}

The authors would like to thank the Referees and the Editor for their interesting comments and suggestions which have contributed to substantially 

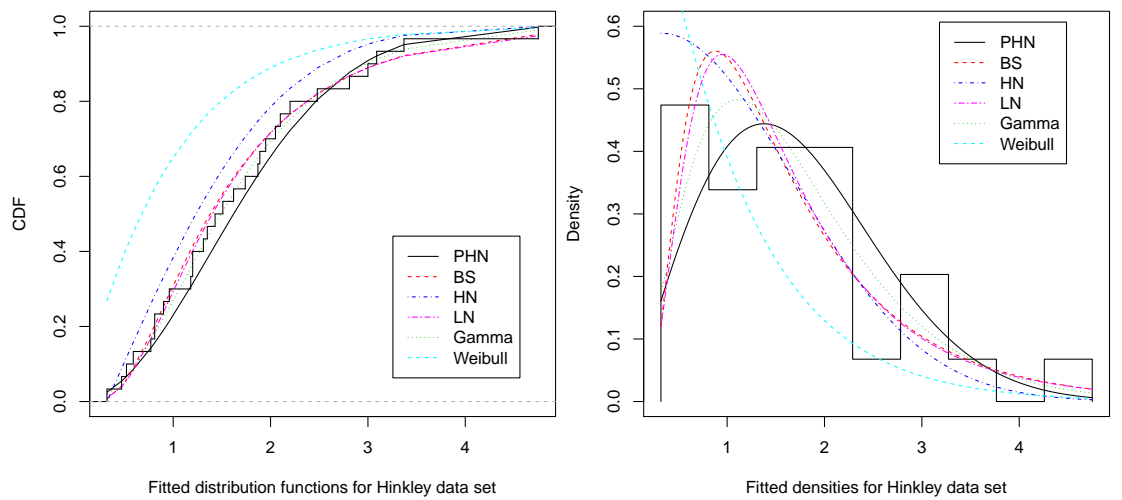

Figure 6. Left panel: Estimated distribution function from the fitted PHN, LN, BS, HN, Weibull and gamma distributions versus the empirical distribution for Hinkley data. Right panel: Estimated densities of the PHN, LN, BS, HN, Weibull and gamma models for Hinkley data.

improving the manuscript. The authors are also indebted to the Yazd University (Yazd, Iran) for support this research.

\section{References}

Aarset, M.V. (1987). How to Identify Bathtub Hazard Rate. IEEE Transactions Reliability, 36, 106-108.

Aigner, D.J., Lovell, C.A.K. and Schmidt, P. (1977). Formulation and Estimation of Stochastic Frontier Production Models. J. Econometrics, 6, 21-37.

Azzalini, A. (1985). A Class of Distributions which Includes the Normal Ones. Scand. J. Statist., 12, 171-178.

Bland, J.M. and Altman, D.G. (1999). Measuring Agreement in Method Comparison Studies. Stat. Meth. Med. Res, 8, 135-160.

Chen, G. and Balakrishnan, N. (1995). A General Purpose Approximate Goodness-of-fit Test. Journal of Quality Technology, 27, 154-161.

Chou, C.Y. and Liu, H.R. (1998). Properties of the Half-normal Distribution and Its Application to Quality Control. Journal of Industrial Technology, 14, 4-7. 
Cooray, K. and Ananda, M.M.A. (2008). A Generalization of the Half-normal Distribution with Applications to Lifetime Data. Commun. Statist. Theor. Meth., 37, 1323-1337.

Daniel, C. (1959). Use of Half-normal Plots in Interpreting Factorial Two-level Experiments. Technometrics, 1, 311-341.

Dobzhansky, T. and Wright, S. (1947). Genetics of Natural Populations X. Dispersal Rates in Drosophila Pseudoobscura. Genetics, 28, 304-340.

Flecher C., Allard, D. and Naveau, P. (2009). Truncated Skew-normal Distributions: Estimation by Weighted Moments and Application to Climatic Data. INRA Research Report 39, Available from: http://www.avignon.inra.fr/content/download/7204/111974/version/1/ file/RR2009-39.pdf.

Gelman, A. (2006). Prior Distributions for Variance Parameters in Hierarchical Models. Bayesian analysis, 1, 515-534

Glaser, R.E. (1980). Bathtub and Related Failure Rate Characterizations. Journal of the American Statistical Association, 75, 667-672.

Gradshteyn, I.S. and Ryzhik, I.M. (2000). Table of Integrals, Series, and Products. Academic Press, San Diego.

Haberle, J.G. (1991). Strength and Failure Mechanisms of Unidirectional Carbon FibreReinforced Plastics Under Axial Compression. Unpublished Ph.D. thesis, Imperial College, London.

Hinkley, D. (1977). On Quick Choice of Power Transformations. The American Statistician, 26, 67-69.

Leslie, H. Miller (1956). Table of Percentage Points of Kolmogorov Statistics. J. Am. Stat. Assoc., 51, 111-121.

Meeusen, W.J. and van den Broeck, J. (1977). Efficiency Estimation from Cobb Douglas Production Functions with Composed Error. Int. Econ. Rev, 8, 435-444.

Mitchell, M.R. and Landgraf, R.W. (1996). Advances in Fatigue Lifetime Predictive Techniques: 3rd Volume, ASTM International.

Nichols, M.D. and Padgett, W.J. (2006). A Bootstrap Control Chart for Weibull Percentiles. Quality and Reliability Engineering International, 22, 141-151.

Pescim, R.R., Demetrio, C.G.B., Cordeiro, G.M., Ortega, E.M.M. and Urbano, M.R. (2009). The Beta Generalized Half-normal Distribution. Comput. Stat. Data Anal., 54, 945-957.

Pewsey, A. (2002). Large-sample Inference for the General Half-normal Distribution. Commun. Statist. Theor. Meth., 31, 1045-1054. 
Pewsey, A. (2004). Improved Likelihood based Inference for the General Half-normal Distribution. Commun. Statist. Theor. Meth., 33, 197-204.

Radson, D. and Alwan, L.C. (1995). Detecting Variance Reductions using the Moving Range. Quality Engineering, 8, 165-178.

Smith, R.L. (1985). Maximum Likelihood Estimation in a Class of Nonregular Cases. Biometrika, 72, 67-90.

Wiper, M.P., Giron, F.J. and Pewsey, A. (2008). Objective Bayesian Inference for the Halfnormal and Half-t distributions. Commun. Statist. Theor. Meth., 37, 3165-3185.

Watson, G.S. (1961). Goodness-of-fit Tests on a Circle. Biometrika, 48, 109-114.

\section{Appendix}

The distributions of Birnbaum-Saunders, log-normal, gamma, Weibull and half-normal used in Tables 5 and 6 are given by:

- Birnbaum-Saunders:

$f(x ; \alpha, \beta)=\frac{1}{2 \sqrt{2 \pi} \alpha \beta}\left(\left(\frac{\beta}{x}\right)^{\frac{1}{2}}+\left(\frac{\beta}{x}\right)^{\frac{3}{2}}\right) \exp \left\{-\frac{1}{2 \alpha^{2}}\left(\frac{x}{\beta}+\frac{\beta}{x}-2\right)\right\}, \quad x>0, \alpha>$ $0, \beta>0$,

- Log-normal:

$f(x ; \mu, \sigma)=\frac{1}{\sqrt{2 \pi} \sigma x} \exp \left\{-\frac{1}{2}\left(\frac{\ln x-\mu}{\sigma}\right)^{2}\right\}, \quad x>0,-\infty<\mu<\infty, \sigma>0$,

- Gamma:

$f(x ; k, \theta)=\frac{1}{x \Gamma(k)}\left(\frac{x}{\theta}\right)^{k} \exp \left(-\frac{x}{\theta}\right), \quad x>0, k>0, \theta>0$,

- Weibull:

$f(x ; \alpha, \sigma)=\frac{\alpha}{\sigma}\left(\frac{x}{\sigma}\right)^{\alpha-1} e^{-\left(\frac{x}{\sigma}\right)^{\alpha}}, \quad x \geqslant 0, \alpha>0, \sigma>0$,

- Half-normal:

$f(x ; \mu, \sigma)=\frac{1}{\sigma} \sqrt{2 / \pi} \exp \left\{-\frac{1}{2}\left(\frac{-x-\mu}{\sigma}\right)^{2}\right\}, \quad x>\mu,-\infty<\mu<\infty, \sigma>0$.

\section{Eisa Mahmoudi}

Department of Statistics,

Yazd University,

Yazd, Iran.

email:mahmoudi@yazd.ac.ir

\section{Reihaneh Lalehzari}

Department of Statistics,

Yazd University,

Yazd, Iran.

email: rlalehzari@gmail.com 


\section{Rahmat Sadat Meshkat}

Department of Statistics,

Yazd University,

Yazd, Iran.

email:r.meshkat@gmail.com 